\title{
Linx
}

Revue des linguistes de l'université Paris X Nanterre

42 | 2000

Approches sociolinguistiques du plan phonique

\section{Le terme " relâchement » en sociolinguistique}

The sociolinguistic term "relaxed"

\section{Françoise Gadet}

\section{OpenEdition}

Journals

Édition électronique

URL : http://journals.openedition.org/linx/742

DOI : $10.4000 /$ linx.742

ISSN : 2118-9692

\section{Éditeur}

Presses universitaires de Paris Nanterre

\section{Édition imprimée}

Date de publication : 1 juin 2000

Pagination : 11-20

ISSN : 0246-8743

Référence électronique

Françoise Gadet, « Le terme « relâchement » en sociolinguistique », Linx [En ligne], 42 | 2000, mis en ligne le 20 juin 2012, consulté le 01 mai 2019. URL : http://journals.openedition.org/linx/742 ; DOI : 10.4000/linx.742

Ce document a été généré automatiquement le 1 mai 2019.

Département de Sciences du langage, Université Paris Ouest 


\title{
Le terme " relâchement » en sociolinguistique
}

The sociolinguistic term "relaxed"

\author{
Françoise Gadet
}

1 Il est certes peu satisfaisant de faire appel à un terme comme "relâchement de la prononciation", fortement ambigu, comme d'ailleurs beaucoup des termes sociolinguistiques. Il renvoie en effet à la fois à un sens sociologique (les variantes relâchées sont celles auxquelles ont recours des locuteurs appartenant à des groupes sociaux défavorisés, ou qu'utilisent les locuteurs dans des situations familières), et à un sens technique de phonétique (on peut appliquer ce terme à des sons ou des séquences requérant un moindre effort articulatoire) ${ }^{1}$. Dans les deux sens, le terme véhicule des connotations dépréciatives, et on ne peut exclure que le second sens soit fortement contaminé par le premier : seraient dits relâchés les énoncés de locuteurs dont la langue n'est pas « tenue ».

2 Bien que consciente des défauts du terme, je continue néanmoins à en faire usage étant donné le manque de concepts et de termes pour aborder toute forme de langue substandard autrement qu'en envers négatif ou désorganisé de la langue standard.

\section{Caractériser les variétés d'un point de vue linguistique}

3 Nous partirons d'une question peu thématisée en sociolinguistique, bien que ses effets indirects reviennent de façon récurrente mais rarement explicite au fil des textes : il s'agit de la caractérisation des variétés (en supposant qu'une telle chose existe). Peuventelles être appréhendées autrement qu'à travers les désignations extra-linguistiques classiques? Les catégories linguistiques en jeu dans une variabilité en direction de la «vernacularité » (terme de Manessy 1993) sont-elles aléatoires dans un système, ou 
possèdent-elles certaines propriétés de langue identifiables qui en feraient de possibles candidats pour ce rôle ? Peut-on généraliser au-delà d'une seule langue?

Cette question peut être dédoublée : 1) étant données deux variantes, est-il possible de reconnaître et de caractériser la variante de prestige (ou la variante vernaculaire) ?; 2) un ensemble de variantes de prestige manifestera-t-il certaines propriétés communes? (de même un ensemble de variantes stigmatisées ?) Une troisième question se profile en toile de fond : 3) en va-t-il de même à tous les niveaux linguistiques ? En particulier, peuton sur une telle question aborder dans les mêmes termes le phonique et le grammatical ?

5 La première question a bien été envisagée, par des phonéticiens. Supposons par exemple les deux variantes [katr] et [kat] (quatre), que l'on présenterait à quelqu'un ne maitrisant le français que de façon scolaire, sans connaissances sur sa variation ; supposons qu'on lui demande laquelle des deux formes correspond à un usage populaire. Il est vraisemblable que notre témoin désignera la deuxième, car le français est loin d'être la seule langue dans laquelle les locuteurs tendent à réduire les groupes consonantiques complexes dans des conditions ordinaires d'utilisation de la langue (voir anglais [laznajt] pour last night). Existe-t-il au contraire des langues dans lesquelles ce serait systématiquement les variantes de prestige qui simplifieraient les groupes complexes? Cela semble peu vraisemblable (Lass 1980, chap. 2) ${ }^{2}$. Et notre exemple n'est sans doute pas isolé : sur l'assimilation ou la tension vocalique, l'expérience donnerait probablement le même résultat.

6 Dans le même esprit, Léon 1973 s'est demandé si l'on pourrait imaginer « que les gens du seizième [arrondissement] parlent avec l'accent faubourien et ceux des faubourgs avec l'accent du seizième » (p. 784).

\section{Des propriétés linguistiques de la vernacularité ?}

7 La deuxième question, concernant les propriétés linguistiques, s'éloigne encore des questionnements de sens commun. Elle n'a à ma connaissance guère été formulée ${ }^{3}$, malgré quelques exceptions comme Kroch 1978, qui se proposait d'étudier l'orientation de la variation phonologique observée à travers l'existence de processus «naturels » de relâchement. Ceux-ci seraient à l'œuvre dans toutes les formes de langue d'un usage ordinaire, comme l'avait déjà supposé Zwicky 1972 en présentant une étude de phénomènes phoniques se manifestant dans le « discours rapide ».

8 Si de tels processus "naturels» devaient être reconnus comme caractéristiques de la vernacularité, comment les nommer? Et comment nommer l'ensemble de phénomènes phoniques, signifiant pour le vernaculaire, qui comporte aussi assimilations consonantiques, coalescences de voyelles, affaiblissements de consonnes intervocaliques...? Pourquoi pas "relâchement»? On peut d'ailleurs noter que des travaux d'un intérêt indéniable dans le champ de la sociolinguistique, non seulement ne se sont pas passés de ce terme, mais en ont fait un élément de leur dispositif interprétatif, comme Guiraud (1969), ou Manessy (1995). On retiendra de plus, pour le conserver, sa mise en œuvre dans des propos courants de locuteurs de variétés dépréciées (par exemple de variétés « régionales» de français, en France ou hors de France) : il n'est pas rare en effet d'entendre ces locuteurs dire qu'ils parlent mal par exemple parce qu'ils «écrasent » ou « avalent » les sons. 
9 Il y a cependant aussi un argument contre l'emploi de ce terme : la difficulté de traduire « relâchement » en une langue autre que le français, où un équivalent même approximatif ne semble pas faire sens, ou apparaît comme technique (« looseness »?, « Nachlassen der Artikulationsspannung » ?). Ce qui laisse entendre que l'on pourrait faire l'économie d'un terme, alors même que des phénomènes semblables interviennent dans ces langues, avec le même type d'enjeu sociolinguistique, mais sans que se soit imposé un terme générique.

\section{Interpréter/expliquer les phénomènes linguistiques en fonction des usages}

Les réflexions de ces deux premiers points s'inscrivent dans un horizon d'explicabilité en sociolinguistique (entreprise dont on connaît la difficulté et les pièges - $c f$. Cameron $1990)$; et nous allons maintenant en envisager les implications.

11 Les «explications $»^{4}$ ici proposées relèvent de deux ordres, interne ou externe. Quand elles sont internes au système, c'est la plupart du temps dans une version fonctionnaliste ou téléologique, caractérisant soit les formes linguistiques, soit la construction grammaticale : moindre effort, facilité de prononciation, naturalité, optimalité... ; ou bien elles sont externes, liées aux modalités d'usage de la langue par le locuteur dans un cadre social. On pourrait identifier une troisième catégorie, avec les «besoins » des locuteurs (Frei 1929), plus ou moins psychologiques (chez Frei : assimilation, différenciation ou clarté, brièveté, invariabilité, expressivité), en fait à cheval sur les deux premières catégories. C'est maintenant surtout du deuxième aspect que nous allons parler, ce qui va nous ramener aux questions de Léon et de Kroch.

Bien que la question de Léon s'appuie sur le jeu de facteurs internes, on ne peut y répondre qu'en se situant dans la perspective de l'usage que différents groupes font de la langue. C'est ainsi qu'il se propose d'interpréter l'attitude phonique présidant aux usages populaires à travers les « bases pulsionnelles de la phonation ", reprises de Fonagy : pour lui, le relâchement y joue un rôle (parmi d'autres phénomènes), mimant dans l'attitude du corps même l'absence de désir d'entrer en communication avec l'autre.

Pour Kroch, ce ne sont pas des raisons psychologiques, mais des considérations d'histoire de l'usage de la langue qui expliquent que certaines formes phoniques aient pu devenir stigmatisées. Etant perçues par les locuteurs comme ordinaires, parce que liées surtout à des situations peu prestigieuses mettant en cause des locuteurs en proximité physique et sociale dans des interactions quotidiennes, ou apparaissant mal adaptées aux exigences de situations socialement élaborées, elles sont évitées par les locuteurs soucieux de leur statut et de leur façon de parler, plutôt d'ailleurs que délibérément sélectionnées par les locuteurs de couches sociales sans prestige (voir plus bas).

14 Aussi bien Léon que Kroch s'appuient sur le niveau phonique : il reste à se demander ce qu'il en est de ces interrogations appliquées à d'autres niveaux linguistiques.

\section{Phonique vs grammatical}

Le courant variationniste, constitué sur l'étude du phonique, ne voit pas d'inconvénient majeur à poser que faits de syntaxe et faits de phonologie peuvent être traités selon les mêmes procédures, malgré tout ce qui a peu à peu été mis au point à ce propos, au moins 
depuis le structuralisme (Hudson 1980) ${ }^{5}$. Pourtant, pour les phénomènes de vernacularité autres que phoniques, en particulier pour la grammaire, il est peu probable que les « explications » que nous venons d'envisager en termes de processus naturels s'avèrent satisfaisantes.

Nous proposons avant tout de distinguer entre deux séries de faits grammaticaux, compte tenu de leur comportement grammatical et sociolinguistique. Nous considérerons d'un côté des phénomènes morphologiques ou syntaxiques "proches de la phonologie" (comme la présence/absence du ne de négation), d'autre part des phénomènes «proches du discours » (comme la dislocation, la subordination, l'interrogation). On regardera les premiers comme n'ayant que des effets locaux (présence/absence d'une unité, ou bien « $\mathrm{x}$ à la place de $\mathrm{y}$ ", sans effet radical sur la signification), et les deuxièmes comme imposant une réorganisation globale.

La première catégorie de phénomènes peut sans doute être abordée dans des termes proches de ceux mis en avant pour le plan phonique. Nous évoquerons ici, plutôt que des exemples archi-répétés, ces faits issus du français québécois ordinaire : [a :mezõ] pour à la maison, [dõmẽ] pour dans la main, [syatab] pour sur la table, mettent en jeu des phénomènes d'ordre morpho-phonologique, que Manessy 1993 qualifie comme "écrasements des mots outils» (p.410). Les autres phénomènes, plus proches de la syntaxe et des effets discursifs, exigeraient qu'il soit fait appel à d'autres ordres d'explication, sémantique, pragmatique ou cognitif. En effet, on ne peut plus ici s'appuyer sur ce qui a fait l'affaire pour le phonique ou pour les phénomènes du premier niveau grammatical, pour lesquels on peut admettre que les variantes non standard sont sémantiquement équivalentes aux variantes standard.

\section{Propriétés du phonique et du syntaxique}

18 Les phénomènes grammaticaux $\mathrm{du}$ premier ordre sont, dans la littérature sociolinguistique, les plus fréquemment décrits (compte tenu de la relative discrétion de la sociolinguistique en matière de syntaxe). Ils ont souvent été abordés sous l'angle du degré de simplicité/complexité, ou de la naturalité. Ceci les rapproche encore des phénomènes phoniques, et contribue à les distinguer des phénomènes grammaticaux du deuxième ordre.

Mais des termes comme «simplicité » ou «naturalité » ne peuvent signifier la même chose selon le niveau linguistique. Ainsi, quand Berruto 1983 veut caractériser « l'italien populaire ", d'un point de vue essentiellement syntaxique, sous l'angle de la complexité (afin de tester l'hypothèse de sens commun selon laquelle le populaire serait plus simple que le standard), il s'efforce avant tout de définir cette notion. Il aboutit d'ailleurs à établir que la simplicité ne suffit pas à définir le populaire (de même Lass 1980, pour la critique d'un fonctionnalisme radical).

La différence ainsi mise en lumière, entre phonique et morphologique d'une part, syntaxique et discursif de l'autre, ne saurait surprendre que certains sociolinguistes qui ont souvent voulu supposer que les mêmes procédures pouvaient s'appliquer à tous les niveaux. Elle ne surprendra pas les phonologues, et c'est à deux d'entre eux que j'emprunte la citation suivante: «The crux of our position is that facts pertaining to the two domains - phonology on the one hand, and syntax and semantics, on the other - are 
of a very different nature and that there is therefore no reason to assume a priori that they must be covered by formally similar theories » (Bromberger \& Halle 1989, p. 52).

Ce que visent ici Bromberger et Halle concerne le plan formel, mais je me propose de prolonger leur réflexion en supposant la même opposition dans la dynamique de la pratique des locuteurs. La réflexion d'Armstrong 1997 me semble suivre la même voie : en reprenant des réflexions de Hudson 1980, il avance l'hypothèse que ce ne seraient pas les mêmes aspects d'une "personnalité sociolinguistique» (ou identité) qui se verront exprimés par des traits variables de la syntaxe ou de la phonologie, les traits phoniques étant davantage liés à des aspects concernant les origines (réelles ou revendiquées) du locuteur, et les aspects grammaticaux le statut personnel qu'il a acquis (par exemple le niveau d'éducation) ${ }^{6}$.

\section{Vernaculaire et standardisation}

Nous avons jusqu'ici fait comme si les produits linguistiques étaient le fruit d'une histoire plus ou moins harmonieuse et naturelle, ce qui n'est évidemment pas le cas dans les sociétés occidentales, dont les langues sont passées par un processus de standardisation qui a filtré. Et justement, le niveau phonique et le niveau syntaxique ne se sont pas historiquement présentés de façon équivalente devant la standardisation : le syntaxique a sûrement été moins standardisé que le morphologique, faute d'être connu et décrit jusque dans son fonctionnement le plus ordinaire, mais il l'est beaucoup plus que le phonique.

La standardisation au niveau phonique recherchera donc seulement la marginalisation des « écarts les plus extrêmes » et la proximité de l'écrit, alors que les enjeux sont plus intriqués en syntaxe. Les registres peu formels semblent manifester plus de dépendance du supra-segmental et du contexte, avec des modes de signification implicites, comme la parataxe ou l'ellipse. Les registres plus formels s'appuient au contraire sur les ressources explicites de la syntaxe (comme le degré d'élaboration) ${ }^{7}$. Ainsi, on a pu opposer (Gadet 1998) les ressources syntaxiquement explicites à l'œuvre dans les relatives de (a) (standard) ou de (b) (populaire), aux ressources discursivement lâches et reposant crucialement sur le contexte de (c) ou de (d) (populaires également, mais surtout relevant de contextes familiers et partagés entre locuteurs socialement proches) :

- (a) ouvre donc le tiroir dans lequel tu l'as mis hier

- (b) elle a un nounours qu'elle peut pas dormir sans

- (c) elle me coûte cher ma salle de bain / que je me sers pas d'ailleurs

- (d) la cinq / c'est la seule qu'ils ont écrit

Quelle que soit la dénomination traditionnellement retenue (qui reflète des caractéristiques sociales ou démographiques des locuteurs, plutôt qu'une caractérisation par la situation ${ }^{8}$ ), on peut supposer que c'est ici de fait le contexte d'usage (en particulier dans quelle mesure la possibilité d'interpréter prend appui sur le contexte immédiat) qui l'emporte sur les particularités sociales de l'énonciateur.

\section{Vernaculaire et surveillance}

Nous terminerons sur un problème que nous ne ferons que soulever : le relâchement estil la contrepartie d'une absence de surveillance de la part du locuteur? C'est plus ou 
moins ce que nous avons laissé entendre jusqu'à présent, et c'est la plupart du temps ce que supposent les réflexions sociolinguistiques en définissant le plan stylistique, dans la foulée de Labov, comme un effet du degré d'auto-surveillance.

Pourtant, il y a de bonnes raisons pour ne pas se satisfaire d'une définition du style sous ce seul angle psychologique (Bell 1984 pour une critique de cette conception, qui dénonce en particulier son absence de fondements empiriques). Par exemple Dressler \& Wodak 1982 distinguent (pour l'allemand de Vienne, mais la même distinction semble adaptable à toute langue standardisée), parmi les formes phoniques de prestige, entre celles qui ne font que contrecarrer un processus naturel de relâchement (exemple en français : ne pas effectuer une assimilation), et celles qui relèvent d'une sélection délibérée comme marques de prestige, réelles ou supposées (effectuer une liaison rare, ou prononcer certaines consonnes graphiques). Les deux séries de phénomènes ne relèvent pas des mêmes démarches de locuteur, et ne sont pas non plus l'image inversée l'une de l'autre. Donc, même pour les phénomènes phoniques, l'explication par l'auto-surveillance est insuffisante ; et cela est encore plus net pour les phénomènes grammaticaux.

Si le facteur surveillance (ou auto-surveillance) semble s'imposer avec l'évidence du sens commun, on pourrait penser à première vue que c'est aussi ce que suppose Manessy quand il écrit : « le lieu de la vernacularité est celui où l'on ne se pose pas de questions » (1993, p. 410). Mais il nous semble en même temps ouvrir une piste autre que psychologique, celle du fonctionnement de la communauté, de la place du locuteur dans sa communauté et de l'appropriation par celui-ci d'une variété de langue, en particulier quand il souligne le caractère identitaire des relâchements, plus nombreux en proximité communautaire : il parle de " haut degré de connivence, aussi bien en ce qui concerne la structure type de l'énoncé que pour l'interprétation des données extralinguistiques de la situation d'énonciation » (1995, p. 129).

Aucune des conclusions vers lesquelles se dirigent ces quelques réflexions ne vaut pleinement pour les domaines autres que le phonique, car si l'on voulait parler, comme pourraient le faire des grammairiens normatifs, de "relâchement de la syntaxe ", ce serait en franchissant une étape supplémentaire dans le métaphorique. Ce qui invite encore à conclure au caractère spécifique, parmi les dimensions du langage, de la prononciation, certainement en relation avec le lien au corps et à sa posture : à côté des hypothèses déjà évoquées de Fonagy, on pensera à la réflexion de Bourdieu (par exemple 1982). Des notions comme l'habitus linguistique ou l'hexis corporelle dépendent trop de l'attitude du corps pour pouvoir renvoyer de façon intéressante à un plan linguistique autre que le phonique.

Il faut donc reconnaître une incontestable affinité entre sociolinguistique et plan phonique (illustrée - avant tout mais pas seulement - par le paradigme variationniste). Mais peut-être est-il temps de tester la validité des hypothèses, et du côté du matériau linguistique en jeu, et dans les ressorts sociolinguistiques observables, en acceptant le défi de sollicitations au-delà de ce champ privilégié qu'est le phonique. 


\section{BIBLIOGRAPHIE}

ARMSTRONG N., 1997, « A sociolinguistic perspective on variable grammar in French and in

English », Revue PArole n³-4, 191-216.

BELL A., 1984, « Language Style as Audience Design », Language in Society n 13, 145-204.

BERRUto G., 1983, «L'italiano popolare e la semplificazione linguistica », Vox Romanica 42, 38-79.

BOURDIEU P., 1982, Ce que parler veut dire, Paris, Fayard.

BRomberger S. \& Halle M., 1989, « Why Phonology is different », Linguistic Inquiry 20-1, 51-70.

CAMERON D., 1990, « Demythologizing sociolinguistics: why language does not reflect society », in J. Joseph \& T. Taylor Eds, Ideologies of language, London and New York, Routledge, 79-93.

CHESHIRE J., 1997, « Involvement in 'standard' and 'nonstandard' English », inTaming the Vernacular: from dialect to written standard language, J. Cheshire and D. Stein (Eds), Harlow, Longman, 68-82.

DRESSLER W. \& WODAK R., 1982, « Sociophonological methods in the study of sociolinguistic variation in Viennese German », Language in Society nำ11, 339-70.

FREI H., 1929, La grammaire des fautes, Genève, Republications Slatkine.

GADET F., 1998, «Cette dimension de variation que l'on ne sait nommer », Sociolinguistica n 12, 53-71.

GUIRAUD P., 1969, « Français populaire ou français relâché ? », Le français dans le monde n 69, 23-7. HUDSON R., 1980, Sociolinguistics, Cambridge University Press.

KRocH A., 1978, « Toward a Theory of Social Dialect Variation », Language in Society n ${ }^{\circ} 7-1,17-36$.

LASS R., 1980, On Explaining Language Change, Cambridge University Press.

LAVANDERA B., 1978, « Where does the Sociolinguistic Variable Stop? », Language in Society $\mathrm{n}^{\circ}$ 7, 171-82.

LÉON P., 1973, « Réflexions idiomatologiques sur l'accent en tant que métaphore sociolinguistique », French Review Vol XLVI n 4, 783-9.

MANESSY G., 1993, « Vernacularité, vernacularisation », in D. de Robillard \& M. Beniamino (Dir), Le français dans l'espace francophone, Paris, Honoré Champion, 407-17.

MANESSY G., 1995, Créoles, pidgins, variétés véhiculaires, Paris, Ed. du CNRS.

ZWICKY A., 1972, « On Casual Speech », inPapers from the Eighth Regional Meeting of the Chicago Linguistic Society, p. Peranteau, J. Levi \& G. Phares (Eds), Chicago Linguistic Society, 607-15.

\section{NOTES}

1. On trouve bien le terme chez des phonéticiens (depuis longtemps, par exemple chez Grammont), bien qu$^{1} \mathrm{il}$ ne soit ni généralisé ni même répandu. 
2. Il n'en a pas toujours été ainsi : jusqu'au $16^{\mathrm{e}}$ siècle, la bonne prononciation omettait le [k] dans dicton, et encore maintenant l'orthoépie conseille de prononcer dompter sans [p].

3. Une hypothèse quant à cette rareté : la question dérange, comme tout ce qui oblige à supposer que les locuteurs ne seraient pas égaux devant les significations dans le discours. Voir Lavandera 1978, l'un des premiers textes à mettre en cause l'homologie des niveaux, en soulignant qu'on ne peut négliger la signification pour ne s'intéresser qu'à des indices, comme le fait le variationnisme dans sa forme classique.

4. Cameron 1990 objecterait ici à juste titre qu'il ne s'agit nullement d'explications au sens logique du terme, seulement de corrélations entre linguistique et social, qui n'expliquent rien mais tout au plus repoussent d'un degré les questions posées, pseudo-explications dont la sociolinguistique s'est bien souvent contentée. On pourrait toutefois considérer qu'il y a là un progrès par rapport à une première tradition dévolue aux anecdotes ponctuelles.

5. Il faudrait tenir compte aussi du rôle joué sur chacune des deux dimensions par le processus de standardisation (voir point 6). Il n'est pas exclu que la différence entre les niveaux que nous défendons ici soit en grande partie un artefact de la standardisation.

6. Il n'est pas difficile de montrer que cette hypothèse n'est pas tout à fait ni toujours vérifiée ; mais nous considérons qu'il est important de formuler une hypothèse, ne serait-ce que pour y opposer des contre-exemples et passer ainsi à davantage d'envergure explicative.

7. Voir Cheshire 1997 sur l'anglais, ou Berruto 1983 sur l'italien, qui tous deux supposent que ce sont les locuteurs favorisés et les situations prestigieuses qui sélectionnent les formes normées, pour des raisons généralement liées à la littéracie. Les autres formes se trouvent ainsi de fait abandonnées aux autres locuteurs et aux autres situations.

8. On voit ici à l'œuvre un trait fréquent de la réflexion sociolinguistique : l'exubérance de dénominations quand le diastratique est en jeu, face à la pauvreté des dénominations pour le diaphasique; il arrive même que n'existent que des dénominations diastratiques pour des nuances de toute évidence diaphasiques. La sociolinguistique s'est effectivement davantage donné pour objectif la caractérisation des locuteurs et des groupes que celle des interactions ou des situations.

\section{RÉSUMÉS}

L'article part du terme "relâchement de la prononciation", dont les sociolinguistes font fréquemment usage en supposant plus ou moins implicitement que les variétés non standard seraient ainsi linguistiquement caractérisables. C'est supposer, sur fond de volonté d'explicabilité des processus sociolinguistiques, que le non-standard se situe du côté du "naturel», en opposition à la « surveillance » que supposerait le standard. On est amené par la discussion de ces propositions à distinguer radicalement entre la syntaxe et la phonologie vis-à-vis des comportements sociolinguistiques.

The article takes as its point of departure the term "relaxed pronunciation". This is a term frequently used by sociolinguists with the more or less implicit assumption that non standard varieties may be characterised in this way. This implies that, assuming that the aim is that sociolinguistic processes should be explicable, non-standard forms might be located on the "natural" end of the spectrum whilst the standard forms are on the "monitored" end. The 
discussion of such assertions leads us to make a radical distinction, where sociolinguistic behaviour is concerned, between syntax and phonology.

\section{AUTEUR}

FRANÇOISE GADET

Université de Paris-X

gadet@u-paris10.fr 